EUROPEAN JOURNAL OF PURE AND APPLIED MATHEMATICS

Vol. 11, No. 1, 2018, 10-22

ISSN 1307-5543 - www.ejpam.com

Published by New York Business Global

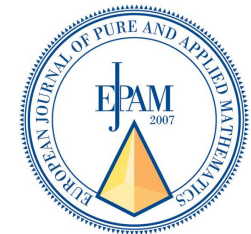

\title{
On ordered hypersemigroups with idempotent ideals, prime or weakly prime ideals
}

Niovi Kehayopulu

To the memory of my teachers Professor Nazım Terzioğlu and Professor Suzan Kahramaner

\begin{abstract}
Some well known results on ordered semigroups are examined in case of ordered hypersemigroups. Following the paper in Semigroup Forum 44 (1992), 341-346, we prove the following: The ideals of an ordered hypergroupoid $H$ are idempotent if and only if for any two ideals $A$ and $B$ of $H$, we have $A \cap B=(A * B]$. Let now $H$ be an ordered hypersemigroup. Then, the ideals of $H$ are idempotent if and only if $H$ is semisimple. The ideals of $H$ are weakly prime if and only if they are idempotent and they form a chain. The ideals of $H$ are prime if and only if they form a chain and $H$ is intra-regular. The paper serves as an example to show how we pass from ordered semigroups to ordered hypersemigroups.
\end{abstract}

2010 Mathematics Subject Classifications: AMS 06F99

Key Words and Phrases: ordered hypersemigroup, ideal, weakly prime, prime, idempotent, semisimple, left regular, intra-regular

\section{Introduction and prerequisites}

In our paper in Semigroup Forum 44 (1992) 341-346 [4] we characterized the ordered semigroup $S$ in which the ideals are idempotent in terms of the ideals of $S$ and we proved that this type of ordered semigroups are the semisimple ordered semigroups. We also proved that the ideals of an ordered semigroup $S$ are weakly prime if and only if they are idempotent and they form a chain. And that the ideals of an ordered semigroup $S$ are prime if and only if they form a chain and $S$ is intra-regular. In the present paper we show the way we pass from ordered semigroups to ordered hypersemigroups. For convenience, we will give some definitions-notations already given in [8-10].

An hypergroupoid is a nonempty set $H$ endowed with an hyperoperation

$\circ: H \times H \rightarrow \mathcal{P}^{*}(H) \mid(a, b) \rightarrow a \circ b$ on $H$ and an operation

$*: \mathcal{P}^{*}(H) \times \mathcal{P}^{*}(H) \rightarrow \mathcal{P}^{*}(H) \mid(A, B) \rightarrow A * B$ on $\mathcal{P}^{*}(H)$ (induced by the operation of $H)$ such that $A * B=\bigcup_{(a, b) \in A \times B}(a \circ b)$ for every $A, B \in \mathcal{P}^{*}(H)$. As the operation "*" depends on the hyperoperation "o", an hypergroupoid $H$ is denoted by $(H, \circ)$. Clearly, $A \subseteq B$ implies $A * C \subseteq B * C$ and $C * A \subseteq C * B$ for any $A, B, C \in \mathcal{P}^{*}(H)$ and $H * H \subseteq H$.

Email address: nkehayop@math.uoa.gr (N. Kehayopulu) 
As in ordered semigroups, for a subset $A$ of an hypergroupoid $H$, we denote by $(A]$ the subset of $H$ defined by

$$
(A]:=\{t \in H \mid t \leq a \text { for some } a \in A\},
$$

and we have the following: $A \subseteq(A]$; if $A \subseteq B$, then $(A] \subseteq(B] ;(H]=H ;((A]]=(A]$. For an hypergroupoid $H$, the following two properties, being obvious, play an essential role in the investigation:

(1) If $x \in A * B$, then $x \in a \circ b$ for some $a \in A, b \in B$.

(2) If $a \in A$ and $b \in B$, then $a \circ b \subseteq A * B$.

If $H$ is an hypergroupoid and $A_{i}, B \in \mathcal{P}^{*}(H), i \in I$, then we have the following:

(1) $\left(\bigcup_{i \in I} A_{i}\right) * B=\bigcup_{i \in I}\left(A_{i} * B\right)$.

(2) $B *\left(\bigcup_{i \in I} A_{i}\right)=\bigcup_{i \in I}\left(B * A_{i}\right)$.

An hypergroupoid satisfying the relation $\{x\} *(y \circ z)=(x \circ y) *\{z\}$ for any $x, y, z \in H$ is called hypersemigroup. Identifying the singleton $\{x\}$ by the element $x$ and the $\{z\}$ by $z$ we can write, for short, $x *(y \circ z)=(x \circ y) * z$. For every $x, y \in H$, we can easily show that $\{x\} *\{y\}=x \circ y$, so instead of writing $\{x\} *(y \circ z)=(x \circ y) *\{z\}$ we can also write $\{x\} *(\{y\} *\{z\})=(\{x\} *\{y\}) *\{z\}$. If $(H, \circ)$ is an hypersemigroup, then the operation "*" on $\mathcal{P}^{*}(H)$ is associative, that is $\left(\mathcal{P}^{*}(H), *\right)$ is a semigroup. So in an expression of the form $A_{1} * A_{2} * \cdots * A_{n}$, where $A_{1}, A_{2}, \cdots, A_{n}$ are nonempty subsets of $H$ and $n \in N=\{1,2, \cdots, n\}$ (the set of natural numbers), we can put parentheses in any expression beginning with some $A_{i}$ and ending in some $A_{j}(i, j \in N)$. If " $\leq$ " is an order relation on an hypergroupoid $H$, we denote by " $\preceq$ " the relation on $\mathcal{P}^{*}(H)$ defined by

$$
\preceq:=\{(A, B) \mid \forall a \in A \exists b \in B \text { such that } a \leq b\} .
$$

So, for $A, B \in \mathcal{P}^{*}(H)$, we write $A \preceq B$ if for every $a \in A$ there exists $b \in B$ such that $a \leq b$. This is a reflexive and transitive relation on $\mathcal{P}^{*}(H)$, that is a preorder on $\mathcal{P}^{*}(H)$. The concept of the ordered groupoid [1] can be naturally transferred to an hypergroupoid as follows: An hypergroupoid $(H, \circ)$ is called an ordered hypergroupoid if there is an order relation " $\leq$ " on $H$ satisfying the property $a \leq b$ implies $a \circ c \preceq b \circ c$ and $c \circ a \preceq c \circ b$ for every $c \in H$ (cf. also [11]) and it is denoted by $(H, \circ, \leq)$. The concept of right (left) ideals of ordered groupoids introduced by Kehayopulu in [2], can be naturally transferred to hypergroupoids as follows: If $(H, \circ, \leq)$ is an ordered hypergroupoid, a nonempty subset $A$ of $H$ is called a right (resp. left) ideal of $H$ if (1) $A * H \subseteq A$ (resp. $H * A \subseteq A$ ) and (2) if $a \in A$ and $H \ni b \leq a$, then $b \in A$, that is if $(A]=A$. A subset of $H$ which is both a right and left ideal of $H$ is called an ideal of $H$. Recall that we have $A * H \subseteq A$ (resp. $H * A \subseteq A$ ) if and only if $a \circ h \subseteq A$ (resp. $h \circ a \subseteq A$ ) for every $a \in A$ and every $h \in H$. A nonempty subset $A$ of an ordered hypergroupoid $H$ is called a subgroupoid of $H$ if $A * A \subseteq A$, equivalently if for every $a, b \in A$ we have $a \circ b \subseteq A$. Clearly, every right (left) ideal of an ordered groupoid $H$ is a subgroupoid of $H$. 


\section{Main results}

Proposition 1. Let $(H, \circ, \leq)$ be an ordered hypergroupoid, $a \leq b$ and $c \leq d$. Then we have $a \circ c \preceq b \circ d$.

Proof. Since $a \leq b$ and $c \in H$, we have $a \circ c \preceq b \circ c$. Since $c \leq d$ and $b \in H$, we have $b \circ c \preceq b \circ d$. Since the relation " $\preceq$ " is transitive on $\mathcal{P}^{*}(H)$, we have $a \circ c \preceq b \circ d$.

Proposition 2. Let $(H, \circ, \leq)$ be an ordered hypergroupoid and $A, B, C$ nonempty subsets of $H$ such that $A \preceq B$. Then we have $A * C \subseteq(B * C]$ and $C * A \subseteq(C * B]$.

Proof. Let $x \in A * C$. Then $x \in a \circ c$ for some $a \in A, c \in C$. Since $A \preceq B$ and $a \in A$, there exists $b \in B$ such that $a \leq b$. Since $a \leq b$, we have $a \circ c \preceq b \circ c$. Since $x \in a \circ c$, there exists $y \in b \circ c$ such that $x \leq y$. We have $x \leq y \in b \circ c=\{b\} *\{c\} \subseteq B * C$, so $x \in(B * C]$. Similarly $C * A \subseteq(C * B]$.

Definition 3. Let $H$ be an hypergroupoid or an ordered hypergroupoid. A nonempty subset $T$ of $H$ is called prime (subset) of $H$ if the following assertion is satisfied:

$$
\text { If } A, B \in \mathcal{P}^{*}(H) \text { such that } A * B \subseteq T \text {, then } A \subseteq T \text { or } B \subseteq T \text {. }
$$

It is called weakly prime if we have the following:

If $A, B$ are ideals of $H$ such that $A * B \subseteq T$, then $A \subseteq T$ or $B \subseteq T$.

Proposition 4. Let $H$ be an hypergroupoid or an ordered hypergroupoid. A nonempty subset $T$ of $H$ is a prime subset of $H$ if and only if

$$
a, b \in H \text { such that } a \circ b \subseteq T \text { implies } a \in T \text { or } b \in T \text {. }
$$

Proof. $\Longrightarrow$. Let $a, b \in H, a \circ b \subseteq T$. Since $\{a\},\{b\} \in \mathcal{P}^{*}(H),\{a\} *\{b\}=a \circ b \subseteq T$ and $T$ is prime, we have $\{a\} \subseteq T$ or $\{b\} \subseteq T$. Then $a \in T$ or $b \in T$.

$\Longleftarrow$. Let $A, B \in \mathcal{P}^{*}(H)$ such that $A * B \subseteq T$ and let $A \nsubseteq T$ and $b \in B$. Take an element $a \in A$ such that $a \notin T$. Since $a \circ b \subseteq A * B \subseteq T$, by hypothesis, we have $a \in T$ or $b \in T$. Since $a \notin T$, we have $b \in T$.

Definition 5. Let $H$ be an hypergroupoid or an ordered hypergroupoid. A nonempty subset $T$ of $H$ is called semiprime if

$$
\text { for any } A \in \mathcal{P}^{*}(H) \text { such that } A * A \subseteq T \text {, we have } A \subseteq T \text {. }
$$

Clearly, the prime subsets are both weakly prime and semiprime.

Proposition 6. Let $H$ be an hypergroupoid or an ordered hypergroupoid. A nonempty subset $T$ of $H$ is semiprime if and only if

$$
\text { for any } a \in H \text { such that } a \circ a \subseteq T \text {, we have } a \in T \text {. }
$$

Proof. $\Longrightarrow$. Let $a \in H$ such that $a \circ a \subseteq T$. Since $\{a\} \in \mathcal{P}^{*}(H)$ and $\{a\} *\{a\}=a \circ a \subseteq T$, by hypothesis, we have $\{a\} \subseteq T$, then $a \in T$. 
$\Longleftarrow$. Let $A \in \mathcal{P}^{*}(H)$ such that $A * A \subseteq T$ and $a \in A$. Since $a \circ a \subseteq A * A \subseteq T$, by hypothesis, we have $a \in T$. Thus $A$ is a subset of $T$, and $T$ is semiprime.

Proposition 7. Let $(H, \circ, \leq)$ be an ordered hypergroupoid. If $A$ and $B$ are ideals of $H$, then the intersection $A \cap B$ is an ideal of $H$ as well.

Proof. First of all, since $A$ is a right ideal and $B$ a left ideal of $H$, we have $A \cap B \neq \emptyset$. Indeed: Take an element $a \in A$ and an element $b \in B(A, B \neq \emptyset)$. Then $a \circ b \subseteq A * H \subseteq A$ and $a \circ b \subseteq B * H \subseteq B$, so $a \circ b \subseteq A \cap B$. As $a \circ b$ is a nonempty set, the set $A \cap B$ is a nonempty subset of $H$. In addition, $(A \cap B) * H \subseteq A * H \subseteq A$ and $(A \cap B) * H \subseteq B * H \subseteq B$, thus $(A \cap B) * H \subseteq A \cap B$. If now $x \in A \cap B$ and $H \ni y \leq x$ then, since $y \leq x \in A$ we have $y \in A$ and, since $y \leq x \in B$ we have $y \in B$, so $y \in A \cap B$. Thus $A \cap B$ is a right ideal of $H$. Similarly, $A \cap B$ is a left ideal of $H$ and so it is an ideal of $H$.

Definition 8. A nonempty subset $A$ of an ordered hypergroupoid $(H, \circ, \leq)$ is called idempotent if $A=(A * A]$.

Theorem 9. Let $(H, \circ, \leq)$ be an ordered hypergroupoid. The ideals of $H$ are idempotent if and only if for any two ideals $A$ and $B$ of $H$, we have

$$
A \cap B=(A * B] .
$$

Proof. $\Longrightarrow$. Let $A, B$ be ideals of $H$. By Proposition $7, A \cap B$ is an ideal of $H$. By hypothesis, we have

$$
\begin{aligned}
A \cap B & =((A \cap B) *(A \cap B)] \subseteq(A * B] \\
\subseteq & (A * H] \cap(H * B] \subseteq(A] \cap(B]=A \cap B .
\end{aligned}
$$

Thus we have $A \cap B=(A * B]$.

$\Longleftarrow$. Let $A$ be an ideal of $H$. By hypothesis, we have $A=A \cap A=(A * A]$, so $A$ is idempotent.

Proposition 10. [10; Lemma 2.8] Let $(H, \circ, \leq)$ be an ordered hypergroupoid and $A, B \in$ $\mathcal{P}^{*}(H)$. Then we have $(A] *(B] \subseteq(A * B]$.

Proposition 11. Let $(H, \circ, \leq)$ be an ordered hypergroupoid and $A, B \in \mathcal{P}^{*}(H)$. Then we have

$$
(A * B]=((A] *(B]]=((A] * B]=(A *(B]] .
$$

Proof. Let us prove the equality $((A] *(B]]=((A] * B]$. The rest of the proposition can be proved in a similar way. First of all, the sets $(A]$ and $(B]$ are nonempty subsets of $H$ as $A$ and $B$ are so. Since $B \subseteq(B]$, we have $(A] * B \subseteq(A] *(B]$, then $((A] * B] \subseteq((A] *(B]]$. Let now $t \in((A] *(B]]$. Then $t \leq u$ for some $u \in(A] *(B]$. We have $u \in x \circ y$ for some $x \in(A], y \in(B]$. Then $x \leq a$ for some $a \in A$ and $y \leq b$ for some $b \in B$. By Proposition 1 , we have $x \circ y \preceq a \circ b$. Since $u \in x \circ y$, we have $u \leq v$ for some $v \in a \circ b$. Then we have $t \leq v \in a \circ b$, then $t \in(a \circ b] \subseteq(A * B] \subseteq((A] * B]$. 
Proposition 12. Let $(H, \circ, \leq)$ be an ordered hypersemigroup and $A, B, C$ nonempty subsets of $H$. Then we have

$$
(A *(B] * C]=(A * B * C] .
$$

Proof. By Proposition 11, we have

$$
\begin{aligned}
(A *(B] * C] & =((A *(B]) * C]=((A * B] * C] \\
& =((A * B) * C]=(A * B * C] .
\end{aligned}
$$

An independent proof is as follows: Let $t \in(A *(B] * C]$. Then $t \leq x$ for some $x \in$ $A *(B] * C, x \in y \circ z$ for some $y \in A *(B], z \in C, y \in u \circ v$ for some $u \in A, v \in(B]$ and $v \leq b$ for some $b \in B$. Then we have

$$
t \leq x \in y \circ z=\{y\} *\{z\} \subseteq(u \circ v) *\{z\} .
$$

Since $v \leq b$, by Proposition 1, we have $u \circ v \preceq u \circ b$. Then, by Proposition 2, we have $(u \circ v) *\{z\} \subseteq((u \circ b) *\{z\}]$. Hence we obtain

$$
t \leq x \in((u \circ b) *\{z\}]=(\{u\} *\{b\} *\{z\}] \subseteq(A * B * C],
$$

and then $t \in((A * B * C]]=(A * B * C]$, so $(A *(B] * C] \subseteq(A * B * C]$. On the other hand, since $B \subseteq(B]$, we have $(A * B * C] \subseteq(A *(B] * C]$.

Let $H$ be an hypersemigroup. For a nonempty subset $A$ of $H$ we denote by $I(A)$ the ideal of $H$ generated by $A$. For $A=\{a\}(a \in H)$, we write $I(a)$ instead of $I(\{a\})$.

Proposition 13. Let $(H, \circ, \leq)$ be an ordered hypersemigroup and $A$ a nonempty subset of $H$. Then we have

$$
I(A)=(A \cup(H * A) \cup(A * H) \cup(H * A * H)] .
$$

Proof. We set $T:=(A \cup(H * A) \cup(A * H) \cup(H * A * H)]$. The set $T$ is a nonempty subset of $H$ containing $A$. Moreover, $T$ is an ideal of $H$. In fact:

$$
\begin{aligned}
T * H & =(A \cup(H * A) \cup(A * H) \cup(H * A * H)] * H \\
& =(A \cup(H * A) \cup(A * H) \cup(H * A * H)] *(H] \\
& \subseteq((A \cup(H * A) \cup(A * H) \cup(H * A * H)) * H] \quad \text { (by Proposition 10) } \\
& =((A * H) \cup(H * A * H) \cup(A * H * H) \cup(H * A * H * H)]
\end{aligned}
$$




$$
=((A * H) \cup(H * A * H)] \subseteq T ;
$$

also $(T]=T$. Similarly $T$ is a left ideal of $H$. Let now $K$ be an ideal of $H$ such that $K \supseteq A$. Then $T \subseteq K$. Indeed, we have

$$
\begin{aligned}
T & =(A \cup(H * A) \cup(A * H) \cup(H * A * H)] \\
& \subseteq(K \cup(H * K) \cup(K * H) \cup(H * K * H)]=(K]=K .
\end{aligned}
$$

Proposition 14. Let $(H, \circ, \leq)$ be an ordered hypersemigroup. If $A$ is a left ideal and $B$ is a right ideal of $H$, then the set $(A * B]$ is an ideal of $H$.

Proof. Since $A$ and $B$ are nonempty subsets of $H$, the set $A * B$ is also a nonempty subset of $H$ and so is $(A * B]$. In addition,

$$
\begin{aligned}
H *(A * B] & =(H] *(A * B] \subseteq(H *(A * B)] \text { (by Proposition 10) } \\
& =((H * A) * B] \subseteq(A * B],
\end{aligned}
$$

similarly $(A * B] * H \subseteq(A * B]$. Let now $x \in(A * B]$ and $H \ni y \leq x$. We have $x \leq u$ for some $u \in A * B$. Since $H \ni y \leq u \in A * B$, we have $y \in(A * B]$. Thus $(A * B]$ is an ideal of $H$.

Corollary 15. If $H$ is an ordered hypersemigroup and $A, B$ ideals of $H$, then the set $(A * B]$ is an ideal of $H$.

This is the concept of semisimple ordered semigroups introduced by Kehayopulu in [7]: An ordered semigroup $(S, \cdot, \leq)$ is called semisimple if for every $a \in S$ there exist $x, y, z \in S$ such that $a \leq x a y a z$. This is equivalent to saying that $a \in(S a S a S]$ for every $a \in S$ or $A \subseteq(S A S A S]$ for any $A \subseteq S$. This concept can be naturally transferred to ordered hypersemigroups by the following definition.

Definition 16. An ordered hypersemigroup $(H, \circ, \leq)$ is called semisimple if for every $a \in H$ there exist $x, y, z \in H$ such that $\{a\} \preceq(x \circ a) *(y \circ a) *\{z\}$.

That is, for every $a \in H$ there exist $x, y, z, t \in H$ such that $t \in(x \circ a) *(y \circ a) *\{z\}$ and $a \leq t$.

Clearly,

$$
\begin{aligned}
(x \circ a) *(y \circ a) *\{z\} & =\{x\} *(a \circ y) *(a \circ z)=(x \circ a) *\{y\} *(a \circ z) \\
& =\{x\} *\{a\} *\{y\} *\{a\} *\{z\} .
\end{aligned}
$$

Proposition 17. Let $(H, \circ, \leq)$ be an ordered hypersemigroup. The following are equivalent:

(1) $H$ is semisimple.

(2) $a \in(H *\{a\} * H *\{a\} * H]$ for every $a \in H$.

(3) $A \subseteq(H * A * H * A * H]$ for every nonempty subset $A$ of $H$. 
Proof. (1) $\Longrightarrow(2)$. Let $a \in H$. Since $H$ is semisimple, there exist $x, y, z, t \in H$ such that $a \leq t \in\{x\} *\{a\} *\{y\} *\{a\} *\{z\} \subseteq H *\{a\} * H *\{a\} * H$, so we have $a \in(H *\{a\} * H *\{a\} * H]$.

$(2) \Longrightarrow(3)$. Let $A \in \mathcal{P}^{*}(H)$ and $a \in A$. By (2), we have

$$
a \in(H *\{a\} * H *\{a\} * H] \subseteq(H * A * H * A * H] .
$$

$(3) \Longrightarrow(1)$. Let $a \in H$. By (3), we have $a \in\{a\} \subseteq(H *\{a\} * H *\{a\} * H]$. Then $a \leq t$ for some $t \in(H *\{a\} * H) *\{a\} * H, t \in u \circ v$ for some $u \in H *\{a\} * H, v \in\{a\} * H$, $u \in w \circ y$ for some $w \in H *\{a\}, y \in H, w \in x \circ a$ for some $x \in H$ and $v \in a \circ z$ for some $z \in H$. Thus we have

$$
\begin{aligned}
t \in u \circ v & =\{u\} *\{v\} \subseteq(w \circ y) *\{v\}=\{w\} *\{y\} *\{v\} \\
& \subseteq(x \circ a) *\{y\} *(a \circ z), \text { where } x, y, z \in H
\end{aligned}
$$

and $a \leq t$, so $H$ is semisimple.

Theorem 18. An ordered hypersemigroup $(H, \circ, \leq)$ is semisimple if and only if the ideals of $H$ are idempotent.

Proof. $\Longrightarrow$. Let $A$ be an ideal of $H$. If $x \in A$ then, since $H$ is semisimple, we have $x \in(H *\{x\} * H *\{x\} * H]$. Then $x \leq t$ for some $t \in(H *\{x\} * H) *(\{x\} * H)$. Then

$$
t \in a \circ b \text { for some } a \in H *\{x\} * H, b \in\{x\} * H .
$$

Since $a \in H *\{x\} * H \subseteq(H * A) * H \subseteq A * H \subseteq A$ and $b \in\{x\} * H \subseteq A * H \subseteq A$, we have $a \circ b \subseteq A * A$. Since $x \leq t \in A * A$, we have $x \in(A * A]$. Let now $x \in(A * A]$. Then $x \leq t$ for some $t \in A * A$. Since $t \in A * A$, we have $t \in a \circ b$ for some $a, b \in A$. Since $a, b \in A$ and $A$ is a subsemigroup of $H$, we have $a \circ b \subseteq A * A \subseteq A$. Since $x \leq t \in A$ and $A$ is an ideal of $H$, we have $x \in A$. Thus the ideals of $H$ are idempotent.

$\Longleftarrow$. Let $a \in H$. By hypothesis, we have $I(a)=(I(a) * I(a)]$. In the implication $(4) \Rightarrow(5)$ of Lemma 2 in [4], we replace the multiplication "." by "*", the proof follows.

Theorem 19. Let $(H, \circ, \leq)$ be an ordered hypersemigroup. The ideals of $H$ are weakly prime if and only if they are idempotent and they form a chain.

Proof. $\Longrightarrow$. Let $A$ be an ideal of $H$. Then $A=(A * A]$. Indeed: By Corollary 15, the set $(A * A]$ is an ideal of $H$. Since $A * A \subseteq(A * A]$ and $(A * A]$ is weakly prime, we have $A \subseteq(A * A] \subseteq(A * H] \subseteq(A]=A$, so $A=(A * A]$. Let now $A, B$ be ideals of $H$. Then $A \subseteq B$ or $B \subseteq A$. Indeed: By Corollary 15, the set $(A * B]$ is an ideal of $H$. Since $A * B \subseteq(A * B]$ and $(A * B]$ is weakly prime, we have $A \subseteq(A * B] \subseteq(H * B] \subseteq(B]=B$ or $B \subseteq(A * B] \subseteq(A * H] \subseteq(A]=A$.

$\Longleftarrow$. Let $T$ be an ideal of $H$ and $A, B$ ideals of $H$ such that $A * B \subseteq T$. Since the ideals of $H$ are idempotent, by Theorem 9, we have $A \cap B=(A * B]$. By hypothesis, we have $A \subseteq B$ or $B \subseteq A$. If $A \subseteq B$, then we have $A=A \cap B=(A * B] \subseteq(T]=T$. If $B \subseteq A$, then $B=A \cap B=(A * B] \subseteq T$, so $T$ is weakly prime. 
This is the notion of an intra-regular ordered semigroup introduced by Kehayopulu in [5]: An ordered semigroup $(S, \cdot, \leq)$ is called intra-regular if for every $a \in S$ there exist $x, y \in S$ such that $a \leq x a^{2} y$, that is if $a \in\left(S a^{2} S\right]$ for every $a \in S$, equivalently if $A \subseteq\left(S A^{2} S\right]$ for every $A \subseteq S$. This concept can be naturally transferred to ordered hypersemigroups in the following definition.

Definition 20. An ordered hypersemigroup $(H, \circ, \leq)$ is called intra-regular if for every $a \in H$ there exist $x, y \in H$ such that $\{a\} \preceq(x \circ a) *(a \circ y)$, that is, for every $a \in H$ there exist $x, y, t \in H$ such that $t \in(x \circ a) *(a \circ y)$ and $a \leq t$.

Instead of writing $(x \circ a) *(a \circ y)$, we can clearly write $\{x\} *(a \circ a) *\{y\}$ or $\{x\} *\{a\} *$ $\{a\} *\{y\}$.

In a similar way as in Proposition 17 we can prove the following proposition.

Proposition 21. Let $(H, \circ, \leq)$ be an ordered hypersemigroup. The following are equivalent:

(1) $H$ is intra-regular.

(2) $a \in(H *\{a\} *\{a\} * H]$ for every $a \in H$.

(3) $A \subseteq(H * A * A * H]$ for every nonempty subset $A$ of $H$.

Proposition 22. If $(H, \circ, \leq)$ is an ordered hypersemigroup then, for every nonempty subset $A$ of $H$, the set $(H * A * H]$ is an ideal of $H$.

Proof. The set $(H * A * H]$ is a nonempty subset of $H$, and we have

$$
\begin{aligned}
(H * A * H] * H & =(H * A * H] *(H] \\
& \subseteq((H * A * H) * H] \quad(\text { by Proposition 10) } \\
& =(H * A *(H * H)] \\
& =(H * A * H] .
\end{aligned}
$$

Similarly, $H *(H * A * H] \subseteq(H * A * H]$, we also have $((H * A * H]]=(H * A * H]$ (as $((X]]=(X]$ holds for any subset $X$ of $H)$.

Theorem 23. Let $H$ be an ordered hypersemigroup. If the ideals of $H$ are weakly prime and semiprime, then they form a chain and $H$ is intra-regular. "Conversely", if the ideals of $H$ form a chain and $H$ is intra-regular, then the ideals of $H$ are prime.

Proof. Suppose the ideals of $H$ are weakly prime and semiprime. Since they are weakly prime, by Theorem 19, they form a chain. Let now $a \in H$. We have

$$
(\{a\} *\{a\}) *(\{a\} *\{a\}) \subseteq(H *\{a\} *\{a\} * H],
$$

where $(H *\{a\} *\{a\} * H]$ is an ideal of $H$. Since the ideals of $H$ are semiprime, we have $\{a\} *\{a\} \subseteq(H *\{a\} *\{a\} * H]$, and $a \in\{a\} \subseteq(H *\{a\} *\{a\} * H]$, so $H$ is intra-regular.

For the converse statement, suppose the ideals of $H$ form a chain and $H$ is intraregular. Since $H$ is intra-regular, the ideals of $H$ are semiprime. In fact: Let $T$ be an ideal 
of $H$ and $a \in H$ such that $a \circ a \subseteq T$. Then

$$
a \in(H *\{a\} *\{a\} * H]=(H *(a \circ a) * H] \subseteq(H * T * H] \subseteq(T]=T,
$$

then $a \in T$, and $T$ is semiprime. Since the ideals of $H$ are semiprime, the following two assertions are satisfied:

(1) $I(A)=(H * A * H]$ for every $A \in \mathcal{P}^{*}(H)$. In fact:

We have $(A * A) *(A * A) \subseteq(H * A * H]$, where $(H * A * H]$ is an ideal of $H$. Since $(H * A * H]$ is semiprime, we have $A * A \subseteq(H * A * H]$, and $A \subseteq(H * A * H]$, so $I(A) \subseteq(H * A * H]$. On the other hand,

$$
(H * A * H] \subseteq(A \cup(H * A) \cup(A * H) \cup(H * A * H)]=I(A),
$$

and condition (1) holds.

(2) $I(x \circ y)=I(x) \cap I(y)$ for every $x, y \in H$. In fact: Let $x, y \in H$. Since $x \circ y \subseteq$ $I(x) * H \subseteq I(x)$, we have $I(x \circ y) \subseteq I(x)$. Since $x \circ y \subseteq H * I(y) \subseteq I(y)$, we have $I(x \circ y) \subseteq I(y)$. Thus we get $I(x \circ y) \subseteq I(x) \cap I(y)$. Let now $t \in I(x) \cap I(y)$. By (1), we have $t \in(H *\{x\} * H]$ and $t \in(H *\{y\} * H]$. Then we have $t \leq u$ for some $u \in H *\{x\} * H$ and $t \leq v$ for some $v \in H *\{y\} * H$. Since $u \in(H *\{x\}) * H$, we have $u \in v \circ b$ for some $v \in H *\{x\}, b \in H$. Since $v \in H *\{x\}$, we have $v \in a \circ x$ for some $a \in H$. Then we have

$$
u \in v \circ b=\{v\} *\{b\} \subseteq(a \circ x) *\{b\}=\{a\} *\{x\} *\{b\}, \text { where } a, b \in H .
$$

Similarly, since $v \in H *\{y\} * H$, we have $v \in\{c\} *\{y\} *\{d\}$ for some $c, d \in H$. Hence we obtain

$$
t \leq u, \text { where } u \in\{a\} *\{x\} *\{b\} \text { for some } a, b \in H
$$

and

$$
t \leq v, \text { where } v \in\{c\} *\{y\} *\{d\} \text { for some } c, d \in H .
$$

Then, by Proposition 1, we have

$$
t \circ t \preceq v \circ u=\{v\} *\{u\} \subseteq\{c\} *(\{y\} *\{d\} *\{a\} *\{x\}) *\{b\} .
$$

On the other hand,

$$
\{y\} *\{d\} *\{a\} *\{x\} \subseteq I(x \circ y)
$$

Indeed, we have

$$
\begin{aligned}
(\{y\} *\{d\} *\{a\} *\{x\}) *(\{y\} *\{d\} *\{a\} *\{x\}) & \subseteq H *\{x\} *\{y\} * H \\
& \subseteq(H *(x \circ y) * H] \\
& =I(x \circ y)(\text { by }(1)) .
\end{aligned}
$$


Since $I(x \circ y)$ is semiprime, we have $(\{y\} *\{d\} *\{a\} *\{x\}) \subseteq I(x \circ y)$. Since $I(x \circ y)$ is an ideal of $H$, we have

$$
\{c\} *(\{y\} *\{d\} *\{a\} *\{x\}) *\{b\} \subseteq H * I(x \circ y) * H \subseteq I(x \circ y) .
$$

Then $t \circ t \preceq v \circ u \subseteq I(x \circ y)$. Again since $I(x \circ y)$ is semiprime, we have $t \in I(x \circ y)$, and condition (2) holds.

We are ready now to prove that the ideals of $H$ are prime. For this purpose, suppose $T$ is an ideal of $H$ and $a, b \in H$ such that $a \circ b \subseteq T$. Since the ideals of $H$ form a chain, we have $I(a) \subseteq I(b)$ or $I(b) \subseteq I(a)$. If $I(a) \subseteq I(b)$ then, by (2), we have

$$
a \in I(a)=I(a) \cap I(b)=I(a \circ b) \subseteq I(T)=T,
$$

so $a \in T$. If $I(b) \subseteq I(a)$, again by (2), we have

$$
b \in I(b)=I(a) \cap I(b)=I(a \circ b) \subseteq T,
$$

so $b \in T$, thus $T$ is prime.

Corollary 24. Let $H$ be an hypersemigroup. The following are equivalent:

(1) The ideals of $H$ are prime.

(2) The ideals of $H$ are weakly prime and semiprime.

(3) The ideals of $H$ form a chain and $H$ is intra-regular.

Remark 25. Here we give some examples of ordered hypersemigroups in which the ideals are idempotent. Following the concept of regular ordered semigroups introduced by Kehayopulu in [3], an ordered hypersemigroup $(H, \circ, \leq)$ is said to be regular if for every $a \in H$ there exists $x \in H$ such that $\{a\} \preceq(a \circ x) *\{a\}(=\{a\} *(x \circ a)=\{a\} *\{x\} *\{a\})$. That is, for every $a \in H$ there exist $x, t \in H$ such that $t \in(a \circ x) *\{a\}$ and $a \leq t$. This is equivalent to saying that $a \in(\{a\} * H *\{a\}]$ for every $a \in H$ or $A \subseteq(A * H * A]$ for every $A \in \mathcal{P}^{*}(H)$. If $H$ is a regular hypersemigroup, then the right ideals and the left ideals of $H$ are idempotent. In fact, let $A$ be a right ideal of $H$. Since $H$ is regular, we have $A \subseteq((A * H) * A] \subseteq(A * A] \subseteq(A * H] \subseteq A$, so $(A * A]=A$. If $A$ is a left ideal of $H$, then we have $A \subseteq(A *(H * A)] \subseteq(A * A] \subseteq(H * A] \subseteq A$, thus $(A * A]=A$. Again following the corresponding notions of ordered semigroups, an ordered hypersemigroup $H$ is called left regular [10] if for every $a \in H$ there exists $x \in H$ such that $\{a\} \preceq\{x\} *(a \circ a)(=$ $(x \circ a) *\{a\}=\{x\} *\{a\} *\{a\})$. That is, for every $a \in H$ there exist $x, t \in H$ such that $t \in\{x\} *(a \circ a)$ and $a \leq t$. This is equivalent to saying that $a \in(H *\{a\} *\{a\}]$ for every $a \in H$ or $A \subseteq(H * A * A]$ for every $A \in \mathcal{P}^{*}(H)$. The left regular ordered hypersemigroups are intra-regular. Indeed, let $A \in \mathcal{P}^{*}(H)$. Then we have

$$
\begin{aligned}
A & \subseteq(H * A * A] \subseteq(H *(H * A * A] * A] \\
& =(H *(H * A * A) * A](\text { by Proposition } 12) \\
& =((H * H) * A * A * A]
\end{aligned}
$$




$$
\subseteq(H * A * A * H]
$$

so $H$ is intra-regular. In intra-regular ordered hypersemigroups the ideals are idempotent. In fact: Let $H$ be an intra-regular ordered hypersemigroup and $A$ an ideal of $H$. Since $A \subseteq(H * A * A * H]$, we have

$$
\begin{aligned}
(A * A] & \subseteq((H * A * A * H] * A]=((H * A * A * H) * A] \text { (by Proposition 11) } \\
& \subseteq(H * A] \subseteq(A]=A \subseteq((H * A) *(A * H)] \subseteq(A * A],
\end{aligned}
$$

then $(A * A]=A$, and $A$ is idempotent. An ordered hypersemigroup $H$ is said to be right regular [10] if for every $a \in H$ there exists $x \in H$ such that $\{a\} \preceq(a \circ a) *\{x\}(=$ $\{a\} *(a \circ x)=\{a\} *\{a\} *\{x\})$. This is equivalent to saying that $a \in(\{a\} *\{a\} * H]$ for every $a \in H$ or $A \subseteq(A * A * H]$ for every $A \in \mathcal{P}^{*}(H)$. The right regular ordered hypersemigroups are also intra-regular. Thus, in left regular, right regular or intra-regular ordered hypersemigroups the ideals are idempotent.

It might be finally mentioned that in commutative ordered hypersemigroups the prime and weakly prime ideals coincide -the proof is the same with the proof of the Proposition in [4], we just have to replace the operation "." of the semigroup by the hyperoperation "o" of the hypersemigroup.

We apply the theorems of the paper to the following two examples.

Example A. We consider the ordered hypersemigroup $H:=\{a, b, c, d, f\}$ defined by the hyperoperation given in the table below and the order below.

\begin{tabular}{c|c|c|c|c|c}
$\circ$ & $a$ & $b$ & $c$ & $d$ & $f$ \\
\hline$a$ & $\{a\}$ & $\{a\}$ & $\{a\}$ & $\{a\}$ & $\{a\}$ \\
\hline$b$ & $\{a\}$ & $\{a, b\}$ & $\{a, b, c, f\}$ & $\{a, b\}$ & $\{a, b, c, f\}$ \\
\hline$c$ & $\{a\}$ & $\{a, b\}$ & $\{c\}$ & $\{a, b, c, f\}$ & $\{a, b, c, f\}$ \\
\hline$d$ & $\{a\}$ & $\{a, b\}$ & $\{a, b, c, f\}$ & $\{a, d\}$ & $\{a, b, c, f\}$ \\
\hline$f$ & $\{a\}$ & $\{a, b\}$ & $\{a, b, c, f\}$ & $\{a, b, c, f\}$ & $\{a, b, c, f\}$ \\
$\leq:=\{(a, a),(a, b),(a, d),(a, f),(b, b),(b, f),(c, c),(c, f),(d, d),(f, f)\}$.
\end{tabular}

We give the covering relation and the figure of $H$.

$$
\prec=\{(a, b),(a, d),(b, f),(c, f)\} .
$$

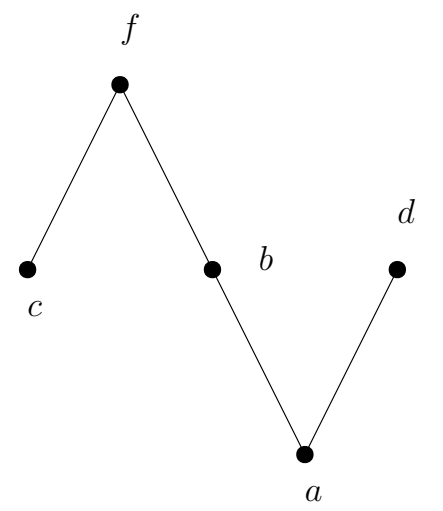


This is intra-regular and the ideals of $H$ are the sets $\{a\},\{a, b, c, f\}$ and $H$ (one can check it) which clearly form a chain. Looking at the table, we immediately see that the ideals of $H$ are prime (that is, $x, y \in H, x \circ y \subseteq\{a\}$ implies $x \in\{a\}$ or $y \in\{a\} ; x, y \in H$, $x \circ y \subseteq\{a, b, c, f\}$ implies $x \in\{a, b, c, f\}$ or $y \in\{a, b, c, f\}$ ), which is also a consequence of Theorem 23.

Example B. We consider the ordered hypersemigroup $H:=\{a, b, c, d, f\}$ defined by the hyperoperation and the figure below:

\begin{tabular}{c|c|c|c|c|c}
$\circ$ & $a$ & $b$ & $c$ & $d$ & $f$ \\
\hline$a$ & $\{b, c\}$ & $\{a\}$ & $\{a\}$ & $\{a\}$ & $\{a\}$ \\
\hline$b$ & $\{a\}$ & $\{b, c\}$ & $\{b, c\}$ & $\{b, c\}$ & $\{b, c\}$ \\
\hline$c$ & $\{a\}$ & $\{b, c\}$ & $\{b, c\}$ & $\{b, c\}$ & $\{b, c\}$ \\
\hline$d$ & $\{a\}$ & $\{b, c\}$ & $\{b, d\}$ & $\{d, f\}$ & $\{d, f\}$ \\
\hline$f$ & $\{a\}$ & $\{b, c\}$ & $\{c\}$ & $\{d, f\}$ & $\{f\}$ \\
& & $b$ & $d$ & & \\
& & $\bullet$ & $\bullet$ & & \\
& & & & & \\
& & & & & \\
& & $\bullet$ & $f$ & &
\end{tabular}

One can check that the ideals of $H$ are the sets $\{a, b, c\}$ and $H$ and that both are idempotent. One can check that for any ideals $A, B$ of $H$, we have $A \cap B=(A * B]$, which is also a consequence of Theorem 9. One can check that $H$ is semisimple, which is also a consequence of Theorem 18. It is obvious that the ideals of $H$ form a chain. So, by Theorem 19, the ideals of $H$ are weakly prime; its independent proof is the following: Let $A, B$ be ideals of $H$ such that $A * B \subseteq\{a, b, c\}$. We have $A=\{a, b, c\}$ or $A=H$ and $B=\{a, b, c\}$ or $B=H$. For $A=B=\{a, b, c\}$, the assumption is obvious. If $A=\{a, b, c\}$ and $B=H$, then $A * B=\bigcup_{\substack{x \in\{a, b, c\} \\ y \in\{a, b, c, d, f\}}} x \circ y=\{a, b, c\}$, again the assumption is obvious. If $A=H$ and $B=\{a, b, c\}$, then $A * B=\{a, b, c, d\} \nsubseteq\{a, b, c\}$, the case is impossible. The case $A=B=H$ is also impossible as $H * H=H$. Moreover, this is an intra-regular hypersemigroup. Since the ideals of $H$ form a chain and $H$ is intra-regular, by Theorem 23, the ideals of $H$ are prime (one can also check it independently).

The Example A has been constructed using the Example 6 in [6] and the Example B using the Example 1 in [5].

Note that we never work directly on an hypersemigroup (ordered hypersemigroup). If we want to obtain a result on an hypersemigroup (ordered hypersemigroup), then we have to prove it first for a semigroup (ordered semigroup) and transfer its proof to hypersemigroup (ordered hypersemigroup). An interesting information concerning the hypersemigroups (without order) will be given in a forthcoming paper. 


\section{References}

[1] G. Birkhoff. Lattice Theory. Amer. Math. Soc. Colloq. Publ. Vol. XXV. Amer. Math. Soc., Providence, R.I. 1967 vi+418 pp.

[2] N. Kehayopulu. Remark on ordered semigroups. Math. Japon. 35(6):1061-1063, 1990.

[3] N. Kehayopulu. On regular duo ordered semigroups. Math. Japon. 37(3):535-540, 1992.

[4] N. Kehayopulu. On prime, weakly prime ideals in ordered semigroups. Semigroup Forum 44(3):341-346, 1992.

[5] N. Kehayopulu. On intra-regular ordered semigroups. Semigroup Forum 46(3):271$278,1993$.

[6] N. Kehayopulu. On regular, intra-regular ordered semigroups. Pure Math. Appl. 4(4):447-461, 1993.

[7] N. Kehayopulu. Characterization of left quasi-regular and semisimple ordered semigroups in terms of fuzzy sets. Int. J. Algebra 6(15):747-755, 2012.

[8] N. Kehayopulu. On hypersemigroups. Pure Math. Appl. (PU.M.A.) 25(2):151-156, 2015.

[9] N. Kehayopulu. On fuzzy prime and fuzzy semiprime ideals in $\leq-$ hypersemigroups. J. Hyperstruct. 5(2):108-114, 2016.

[10] N. Kehayopulu. Left regular and intra-regular ordered hypersemigroups in terms of semiprime and fuzzy semiprime subsets. Sci. Math. Jpn. 80(3):295-305, 2017.

[11] B. Pibaljommee, B. Davvaz. Characterizations of (fuzzy) bi-hyperideals in ordered semihypergroups. J. Intell. Fuzzy Systems 28(5):2141-2148, 2015.

Article Submission Date: O7 June 2017. 